\title{
Structural and kinematic analysis of a stirred tank planetary drive
}

\author{
Alexander A. Prikhodko* \\ Kuban State Technological University, Department of Land transport and mechanics, 350072 \\ Krasnodar, Russia
}

\begin{abstract}
Stirred tanks are used in many industries to intensify various physical and chemical processes. Currently, one of the most promising areas of research is the creation of rotationally reciprocating stirred tanks (RRST), which realize high mixing efficiency due to the difference in the velocities of the stirred liquid. As the actuator of RRST, we proposed to use a planetary gear with elliptical gears. Due to the variable transmission ratio of elliptical gearwheels, the rotationally reciprocating motion of the output shaft is ensured with rotational motion of the input shaft. We conducted a structural analysis of the planetary gear by means of the structural mathematical model of mechanisms and machines. A kinematic model of planetary gear has been constructed and studied, resulting in velocity analogue function of the mechanism output shaft.
\end{abstract}

\section{Introduction}

Stirred tanks are one of the most common types of equipment used to implement various physicochemical processes $[1,2]$. These machines are widely used in chemical, petrochemical, food, construction and many other industries. The disadvantage of currently used devices is the constant angular velocity of the impeller, which often does not allow to provide the required intensity of mixing [3].

Recent studies show that the use of stirrers with nonstationary operating regimes of the impellers intensifies heat and mass transfer in the reactor $[4,5]$. There are many designs of non-standard drives [6-8]; their actuating mechanisms allow implementing different types of motion of the impeller:

- plane-parallel motion (planetary mixers);

- complex spatial motion;

- reciprocating motion;

- rotational motion with an intra-cycle change of the angular velocity;

- rotationally reciprocating motion.

The most promising of these are rotationally reciprocating stirred tanks [6, 9-11]. They provide a high gradient of the stirred liquid velocities, and the use of standard seals enables their integration into existing technological equipment, which can significantly reduce the cost of introducing new machines into the industry.

\footnotetext{
* Corresponding author: sannic92@gmail.com
} 
There is proposed $[10,11]$ a stirred tank in which the reciprocating rotational motion is provided due to the reversible motion of the stepper motor. Such design solution allows experiments in laboratory reactors, but its use on an industrial scale is not rational, since the stepper motor has a low efficiency and cannot be used at a high oscillation frequency of the impeller. To eliminate this disadvantage, a drive scheme for a stirred tank was proposed [12] (Fig. 1), in which the rotational motion of the motor is transformed by the actuator (Fig. 2) into the reciprocating-rotational motion of the impeller.

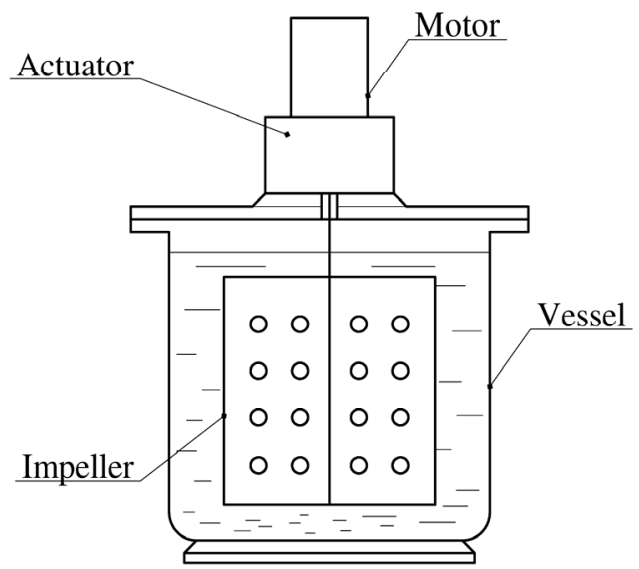

Fig. 1. Scheme of the stirred tank drive.

The planetary actuator consists of an input shaft 1 , a carrier 2 , an output shaft 3 , a sun wheel 4, an elliptical gear 5 on the output shaft and a satellite consisting of a cylindrical gear 6, an elliptical wheel 7 and a shaft 8 . The return-rotational movement of the output shaft is provided by the variable gear ratio of the elliptical gears.

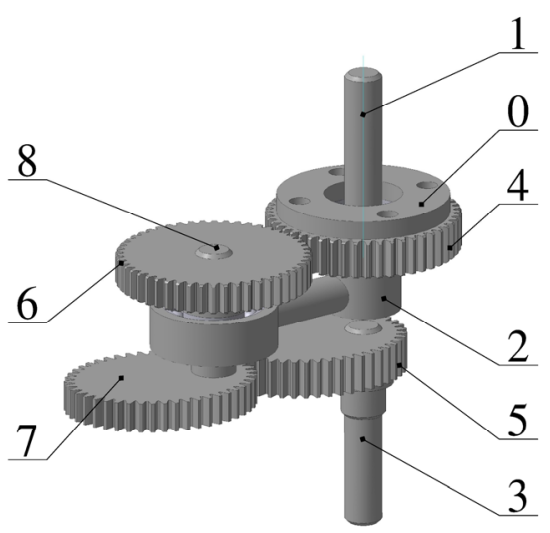

Fig. 2. Construction of actuating mechanism.

As we can see from Fig. 2, in planetary mechanism there is a pair of elliptical gearwheels with a center at the focus of a pitch ellipse. Therefore, it is difficult for balance [13], since the mass centers of the elliptical wheels do not lie on the shaft axes of rotation. To eliminate this drawback, it was proposed to add a pair of modified elliptical wheels with a rotation axes at the centers of symmetry $[14,15]$ to mechanism scheme (Fig. 3). 


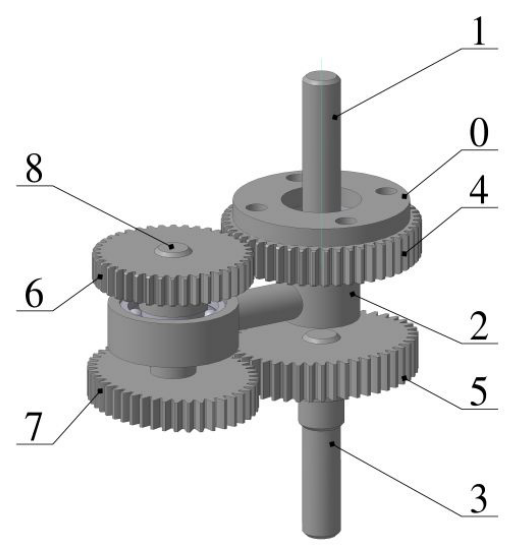

Fig. 3. Planetary mechanism with a pair of modified elliptical gearwheels.

One of the initial stages in the design and calculation of mechanisms is the study of their structure and kinematics. Therefore, the purpose of this work is the structural and kinematic analysis of the proposed planetary gear.

\section{Structural analysis of the mechanism}

Investigation of the structure of the planetary mechanism is carried out using structural mathematical model proposed in [16]:

$$
\left\{\begin{array}{l}
p=\frac{1}{2}\left(\sum_{t=T-j}^{2} t n_{t}+S\right) \\
n=\sum_{t=T-j}^{2} n_{t} \\
W=\sum_{i=1}^{\Pi-1} i p_{i}-k \Pi \\
k=p-n \\
p=\sum_{i=1}^{\Pi-1} p_{i} \\
T \leq k+1
\end{array}\right.
$$

where $n$ is the total number of movable links; $n_{t}$ is the number of movable links with $t$ vertices; $p$ is the total number of kinematic pairs; $p_{i}$ is the number of kinematic pairs of the $i$-th mobility; $T$ is the number of vertices of the base link; $k$ is the number of independent closed circuits; $\Pi$ is the mobility of the space in which the mechanism is synthesized; $W$ is the mobility (DOF) of the mechanism; $S$ is the number of connections to the racks; $i, j$ are integer indices.

The structural scheme of the mechanism includes (Fig. 4): three lower single-moving kinematic pairs $A, C, E\left(p_{1}=3\right)$, two higher two-moving kinematic pairs $B, D\left(p_{2}=2\right)$, one three-vertex link $2\left(n_{3}=1\right)$, two two-vertex links 1 and $3\left(n_{2}=2\right)$. The investigated mechanism exists in a three-moving space $(\Pi=3)$ and has three connections to the rack $(S=3)$. 


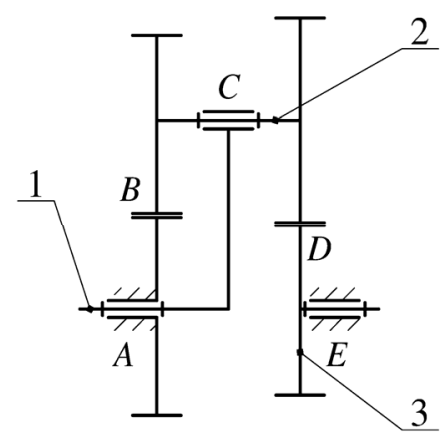

Fig. 4. Structural scheme of the mechanism.

Substituting the values into the structural mathematical model (1), we obtain:

$$
\left\{\begin{array}{l}
5=\frac{1}{2}(3 \cdot 1+2 \cdot 2+3) \\
n=1+2=3 \\
1=1 \cdot 3+2 \cdot 2-2 \cdot 3 \\
k=5-3=2 \\
p=3+2=5 \\
T \leq 3
\end{array}\right.
$$

According to the system of equations (2), the resulting scheme is a 1-DOF mechanism $(W=1)$, which can be used in the drive system of a reciprocating rotational stirred tank.

\section{Kinematic analysis of the planetary mechanism}

To find the analogue of the planetary mechanism output shaft velocity, we construct the plan of its links linear velocities in one of the intermediate positions [17] (Fig. 5).
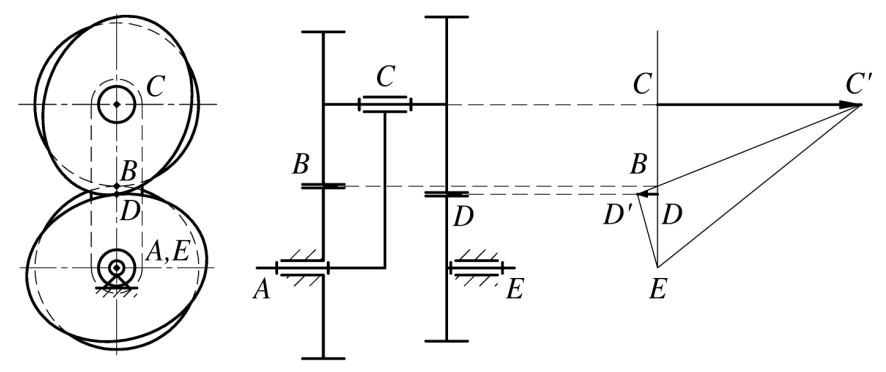

Fig. 5. Plan of the mechanism links linear velocities.

According to Figure 5, the analogue of the output shaft angular velocity is determined by the following equation:

$$
\varphi_{3}^{\prime}=\frac{d \varphi_{3}}{d \varphi_{1}}=\frac{\omega_{3}}{\omega_{1}}=\frac{v_{D} \cdot A C}{v_{C} \cdot D E}=\frac{D D^{\prime} \cdot A C}{C C^{\prime} \cdot D E}=\frac{B D \cdot A C}{B C \cdot D E},
$$


where $\varphi_{3}, \omega_{3}$ are the angle of rotation and the angular velocity of the output shaft $3 ; \varphi_{1}$, $\omega_{1}$ are the angle of rotation and the angular velocity of the input shaft 1 .

The lengths of the segments in equation (3) can be expressed as follows:

$$
\begin{aligned}
& A C=2 R ; \\
& B C=A C=R ; \\
& B D=R-C D ; \\
& D E=A C-C D=2 R-C D,
\end{aligned}
$$

where $R$ is the radius of the cylindrical wheels.

As can be seen from Figure 5, the point $D$ changes its position relative to point $B$, at the same time the direction and modulus of the velocity vector $D D^{\prime}$ changes. At the time when the points $B$ and $D$ are the same, the output shaft of the mechanism is stopped. In order to determine the length of the segment $C D$, we write the equation of the centrode of the elliptical gearwheel with the axis of rotation at the center of symmetry [18]:

$$
C D=\rho=\frac{b}{\sqrt{1-e^{2} \cos ^{2} \varphi}}
$$

where $b$ is the semi-minor axis of the elliptical wheel, $e$ is the eccentricity, $\varphi=\varphi_{1}+\frac{\pi}{2}$ is the angle of rotation of the driving wheel.

Substituting (4)-(8) in (3), we obtain an equation for determining the analogue of the output shaft angular velocity:

$$
\varphi_{3}^{\prime}=\frac{2 R(R-\rho)}{R(2 R-\rho)}=1-\frac{\rho}{2 R-\rho} .
$$

As an example, we consider a planetary mechanism with the following dimensions: $R=22.5 \mathrm{~mm}, a=25 \mathrm{~mm}, b=20 \mathrm{~mm}$. Applying the equation (9), we construct the function of the mechanism velocity analogue (Figure 6).

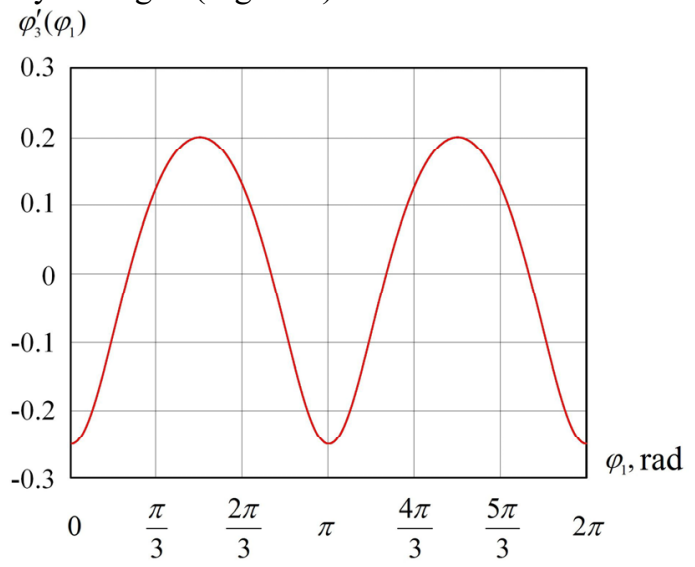

Fig. 6. Function of the mechanism velocity analogue. 
As we can see from Fig. 6, the analogue of the output shaft velocity changes its sign. Thus, at a constant angular velocity of the input shaft, the output shaft with the impeller of the stirred tank will perform a reciprocating-rotational motion.

\section{Conclusion}

We proposed to use the planetary mechanism with elliptical gearwheels as an actuating mechanism of the reciprocating rotational stirred tank. Structural analysis of the obtained device showed that it is a 1-DOF mechanism, which has a correct structure.

We investigated the kinematics of the planetary mechanism; as a result we obtained the function of the mechanism output shaft velocity analogue. Kinematic analysis showed that the developed planetary drive transforms the rotational motion of the input shaft into a reciprocating-rotational motion of the output shaft and can be used as an actuator of the stirred tank.

The reported study was funded by RFBR according to the research project № 18-31-00256.

\section{References}

1. L.D. Schmidt, The Engineering of Chemical Reactions (Oxford University Press, USA, 1998)

2. P.J. Cullen, Food mixing: Principles and applications (Wiley-Blackwell, New York, 2009)

3. A.A. Prikhodko, A.I. Smelyagin, Proceedings of 2015 International Conference on Mechanical Engineering, Automation and Control Systems, 7414910 (2016)

4. J. Kamieński, R. Wójtowicz, Chem. Eng. Process., 42, 1007-1017 (2003)

5. T. Date, Y. Komoda, H. Suzuki, et. al., J. Chem. Eng. Jpn, 51, 159-165 (2018)

6. A.A. Prikhodko, A.I. Smelyagin, Vibroengineering Procedia, 8, 102-107 (2016)

7. N.N. Torubarov, R.M. Malyshev, A.V. Kolebanov, et al., Chem. Petrol. Eng., 52, 327331 (2016)

8. N.N. Torubarov, S.E. Malinin, O.S. Shapovalova, et.al., Chem. Petrol. Eng., 53, 3-9 (2017)

9. A.A. Prikhod'ko, A.I. Smelyagin, Chem. Petrol. Eng., 54, 150-155 (2018)

10. S. Senda, Y. Komoda, Y. Hirata, et. al., J. Chem. Eng. Jpn, 47, 151-158 (2014)

11. S. Senda, Y. Komoda, Y. Hirata, et. al., J. Chem. Eng. Jpn, 49, 341-349 (2016)

12. A.A. Prikhodko, A.I. Smelyagin, J. Phys. Conf. Ser., 858, 012026 (2017)

13. A.A. Prikhodko, A.I. Smelyagin, Engineering and Automation Problems, 4, 62-67 (2016)

14. F.L. Litvin, A. Fuentes, Gear geometry and applied theory (Cambridge University Press, 2004)

15. A.A. Prikhodko, A.I. Smelyagin, A.D. Tsybin, Procedia Engineer, 206, 380-385 (2017)

16. A.I. Smelyagin, A.A. Prikhod'ko, Journal of Machinery Manufacture and Reliability, 45, 500-505 (2016)

17. A.A. Prikhodko, A.I. Smelyagin, Procedia Engineer, 129, 87-92 (2015)

18. H.S.M. Coxeter, Introduction to Geometry (Wiley, New York, 1969) 